\title{
Wavelet based de-noising for on-site partial discharge measurement signal
}

\author{
A. Z. Abdullah', M. Isa ${ }^{2}$, S. N. M. Arshad ${ }^{3}$, M. N. K. H. Rohani ${ }^{4}$, H. S. A. Halim ${ }^{5}$, \\ A. N. Nanyan ${ }^{6}$, H. A. Hamid ${ }^{7}$ \\ 1,2,3,4,6,7 High Voltage Transient \& Insulation Health (HVTrans) Group, Centre of Excellence for Renewable Energy, \\ School of Electrical System Engineering, Universiti Malaysia Perlis (UniMAP), 02600 Arau, Perlis, Malaysia \\ ${ }^{5}$ TNB Research Sdn.Bhd, Research Institution Area, 43000 Kajang, Selangor, Malaysia
}

\begin{tabular}{|c|c|}
\hline Article Info & ABSTRACT \\
\hline Article history: & This paper presents, wavelet based de-noising technique for on-site partial \\
\hline Received Jan 28, 2019 & $\begin{array}{l}\text { discharge (PD) measurement signal. The signal is measured from medium } \\
\text { voltage power cable at } 11 \mathrm{kV} \text { distribution substation. The best mother wavelet. }\end{array}$ \\
\hline Revised Apr 6, 2019 & decomposition level and the type of threshold for the de-noising technique are \\
\hline Accepted May 2, 2019 & $\begin{array}{l}\text { selected based on the signal to noise ratio (SNR) aggregation. The SNR } \\
\text { aggregation is determined based on the minimum, maximum, mean and }\end{array}$ \\
\hline Keywords: & applied for two different PD signals and the selection parameters are done \\
\hline De-Noising & based on the accuracy of de-noising analysis. The analysis is performed in \\
\hline Measurement & $\begin{array}{l}\text { MATLAB software environment and Daubechies } 2(\mathrm{db} 2) \text { is found as the best } \\
\text { mother wavelet at tenth decomposition levels with soft threshold type. This }\end{array}$ \\
\hline On-Site & study is specifically performed to develop the de-noising procedure for on-site \\
\hline Partial Discharge & PD measurement. Overall results indicate that the right selection of the de- \\
\hline Wavelet Transform & $\begin{array}{l}\text { noising procedure will help to improve the PD signal detection from on-site } \\
\text { measurement. }\end{array}$ \\
\hline
\end{tabular}

\section{Corresponding Author:}

A. Z. Abdullah,

School of Electrical System Engineering,

Universiti Malaysia Perlis (UniMAP),

Kampus Pauh Putra, 02600 Arau, Perlis, Malaysia.

Email: zaidiabdullah@unimap.edu.my

\section{INTRODUCTION}

During the last 10 years, the power demand has increased rapidly due to industrialization. In electrical power markets, the reliability of power systems is one of the most important interests of customers and they are willing to pay higher prices to have uninterrupted service. Thus, the utilities attempt to keep their systems safe. Power utilities providers also need to follow the rapid increment of power due to higher demand of electricity. In meeting these higher electricity demands, there have been high power supply interruption reported due to transformer, cable, jointing etc failures. Regarding the IEEE Gold Book on Electrical Reliability, it shown that the highest electrical failures are cause by insulation breakdown [1]. Cable insulation failure were found to be the main causes, and partial discharge (PD) is one of the major causes of insulation degradation in power cable $[2,3]$. PD is a localize discharge that only partially bridges the insulation between the conductor and which may or may not occur adjacent to a conductor [4].

Condition-based maintenance of cable is an important prerequisite to improve reliability of electrical power distribution networks. Traditionally, power cable condition assessment has been performed through off-line diagnostic test such as off line PD test. But performing offline tests require a shut down to the cable under test, and this will require network configuration via multiple switching of switchgears. This problem can 
be minimized by applying on-line PD measurement [5-7]. However, online PD measurement normally has a major problem due to electromagnetic interference (EMI).

Wavelet transform is an analysis tool that can be used for de-noising PD signals from environment interference. While Discrete Wavelet Transform (DWT) is a process taken for PD signals at different times and frequency resolutions [8]. The measured PD signals on-site are transient and non-periodic signals are difficult to characterize by conventional function [9]. For this work, the relevant wavelet transform is selected to analyses and extract the relevant signal and noise in the time domain, while at same time able to improve the signal-to-noise ratio (SNR) [10] based on the signal characteristics.

Theory and advantages of wavelet have been discussed by [11-14]. The parameters applied to wavelets to de-noise a signal can be simply by choosing the best mother wavelet then selecting the threshold. When selecting the mother wavelet, it first identifies the most similar shape of PD signal waveform. Thus, a large wavelet coefficient at few levels is associate with PD signal. Normally noise is widely spread among the level. Therefore, de-noising is performed by reconstructing the signal by eliminating the coefficient combined with noise. The one most similar one to the PD signal shape is selected from the mother wavelet analysis facilities the de-noising process and PD identification. The basic wavelet transform is defining as;

$$
\varphi_{\tau, s}=\frac{1}{\sqrt{s}} \varphi\left(\frac{t-\tau}{s}\right)
$$

Where is the basic function of mother wavelet and are the translation and scale parameter.

De-noising is a technique used to preserve important signals while removing noise [15-17]. The basic idea behind wavelet de-noising is that the wavelet transforms leads to a sparse representation for many realworld signals. This means that the wavelet transforms concentrate signal features in a few large-magnitude wavelet coefficients. Wavelet coefficients which are small in value are typically noise that is removeable without affecting the signal. De-noising the PD signal and noises comprises of three stages namely the application of wavelet transforms, determining the reloaded levels and coefficients, and separation of signal from the noises and its back transformation to rebuild the original PD signal [18].

In current literature, Symlet and Daubechies are the most preferable mother wavelet use in de-noising, however these type of mother wavelet are very common and no previous work in real PD measurement [19]. This paper presents the wavelet based de-noising for on-site partial discharge measurement signal, where PD signals are recorded by using Rogowski Coil (RC) and then MATLAB coding are used to de-noise measured PD signal. In general, electrical partial discharge diagnostics uses pulse and high frequency current induced by PD in an electrical PD measurement circuit $[20,21]$. RC is a sensor which can detect a PD signal based on the magnetic field intensity of the measured signal [22]. In this work, a real pure PD signal is required from the detected signal that combine with noise signal to identify the real condition of the cable.

\section{RESEARCH METHODOLOGY}

In this work, the Rogowski Coil (RC) is used as a measuring sensor for PD measurement. The PD signal is measured together with the noise from on-site measurement. Figure 1 (a) and (b) shows the on-site PD measurement setup for XLPE cable using RC sensor and the high frequency oscilloscope is used for data acquisition connected using $3 \mathrm{~m}$ coaxial cable. The environment in substation are expected producing noise such as from transformer, low voltage equipment, ring main unit and surrounding [23, 24]. Therefore, denoising procedure was performed to determine the pure PD signal.

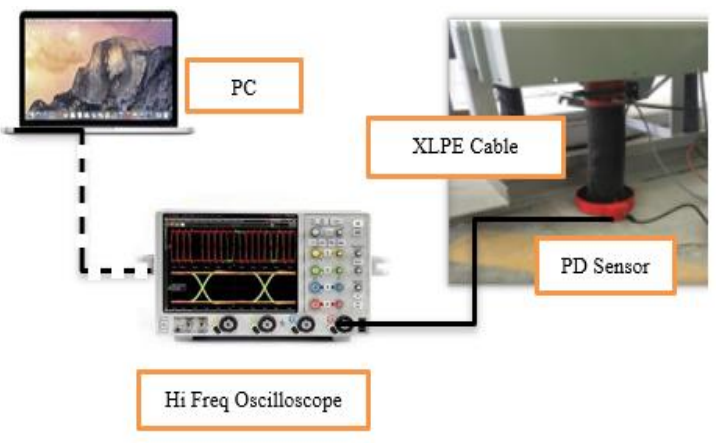

(a)

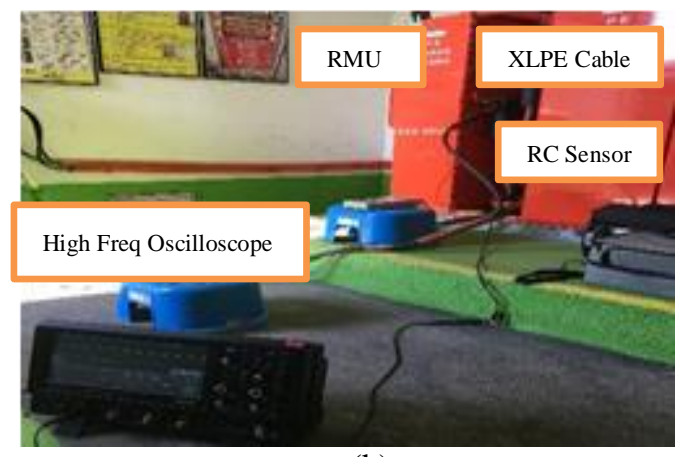

(b)

Figure 1. Test setup of on-line PD measurement using RC at actual power utility substation 
The environmental noise normally including radio waves, power frequency, corona, transient, lightning, thermal noise, harmonic and electrostatic discharge. It also well known that environmental noise present at site can cause distortion of the measured signal and sometimes a small PD signal can be drowned out by the background of high noise. Therefore, noise elimination from PD signal or de-noising is an important for any on-site online PD measurement and analysis.

Figure 2 shows the measured signal from on-site measurement, which measured at $11 \mathrm{kV}, 3$ Phase XLPE underground power cable using Rogowski coil (RC) sensor. The measured signal is contains PD and noise signals [25]. Signal is recorded with $40 \mathrm{kS} / \mathrm{s}$ according to the time domain properties, the noise obtained during on-site online PD measurement in cable can be classified into three categories that are sinusoidal continuous noise, white Gaussian noise and stochastic pulse-shaped noise [26-28]. The procedure of de-noising is schematically shown in Figure 3. Following [25, 26], the noises are removed through the application of the wavelet transformation. This indicate that de-noising is suitable for representation of the noises which are available in the environment. Sometimes noises have amplitudes higher than the partial discharge signal amplitude.

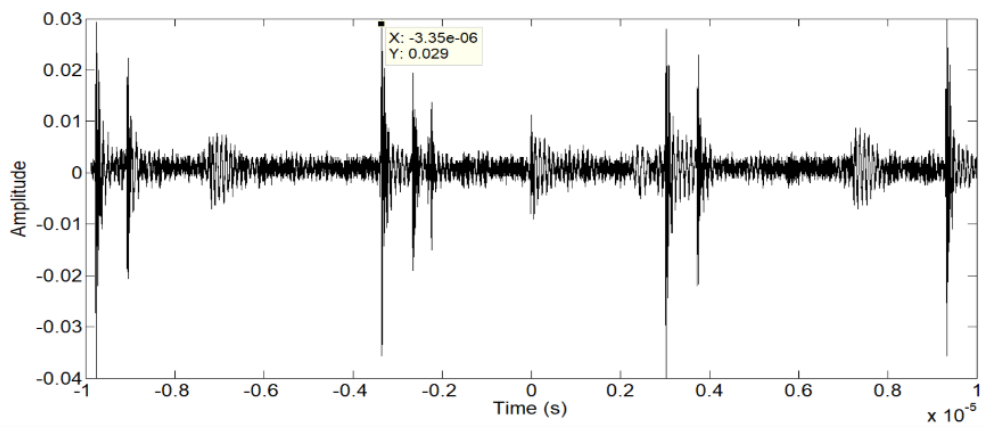

Figure 2. PD signal measured from on-site measurement

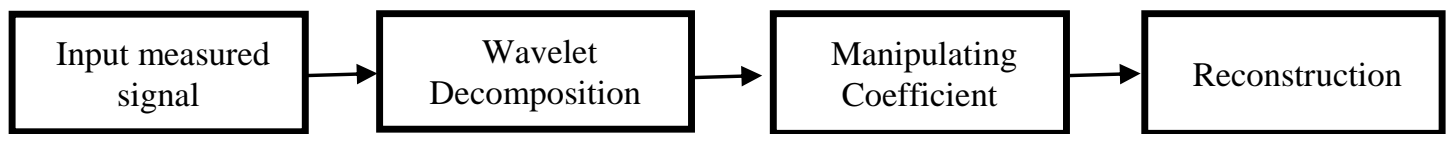

Figure 3. De-noising procedure for PD signal using wavelet transformation

In this research, three major wavelets are chosen namely Symlets, Daubechies and Coiflets. The denoising work is performed under DWT where it comprises of three procedures under standard technique such as threshold variation, decomposition and reconstruction. These procedures play a major role in de-noising PD signal. In DWT, there are two types of thresholds such as soft and hard which are able to remove white noise with little distortion. In this research, soft thresholding is selected since it is an extension of hard thresholding which able to avoid the discontinuity signal. Then the desired level for decomposition and reconstruction is selected by computation in MATLAB. Throughout this research, 10th mother wavelet in Symlets family, 10th mother wavelet Daubechies family and 5th mother wavelet Coiflets family are selected. Families higher than these shows that the PD signal is weaker and mixed with noise. The performance of the de-noising based on mother wavelet was analysed by comparing the signal to noise ratio (SNR). The SNR is calculated using Equation 1.

$$
\operatorname{SNR}(d B)=10 \log \left(\frac{\text { signal }}{\text { noise }}\right)
$$

During de-noising procedure, there were four analysis performed to determine which parameter would be able to produce the best de-noising result. There are maximum, minimum, mean and standard deviations of values measured signal compared to noise. The highest value of SNR indicates the best parameter for wavelet analysis. Then comparison between level of decomposition and type of mother wavelet are compared to determine which the method to use for this research is. 


\section{RESULTS AND ANALYSIS}

There are three mother wavelets use in this paper to de noise measured PD signal combines with the noise. Since noise elimination is an important task in online PD measurement, a clean PD signal from noise was discovered by implementing denoise algorithm. The performance mother wavelet is determined by comparing the SNR values. Table1, Table 2 and Table 3 below shows the comparison of SNR for Symlet, Daubechies and Coiflets. The de-noised signal is shown in the Figure 4 to Figure 9.

Table 1. Comparison result SNR of de-noising signal 1 by symlets wavelet

\begin{tabular}{lcccc}
\hline LEVEL & MAX $(\mathrm{dB})$ & MIN $(\mathrm{dB})$ & MEAN $(\mathrm{dB})$ & STD DEV $(\mathrm{dB})$ \\
\hline SYM1 & 151.7097 & -28.3826 & 1.131428 & 7.903745 \\
SYM2 & 43.70889 & -49.6904 & 0.978222 & $\mathbf{6 . 9 7 0 0 7 7}$ \\
SYM3 & $\mathbf{5 6 . 0 9 0 0 6}$ & -51.6243 & 0.982265 & 6.96467 \\
SYM4 & 45.037 & -44.5719 & $\mathbf{0 . 9 8 6 1 2 5}$ & 6.95752 \\
SYM5 & 42.67066 & $\mathbf{- 3 9 . 4 6 9 8}$ & 0.851026 & 6.874993 \\
SYM6 & 48.69816 & -45.5527 & 0.952668 & 6.950168 \\
SYM7 & 46.27777 & -48.7942 & 0.832591 & 6.826239 \\
SYM8 & 51.46609 & -60.4862 & 0.905391 & 6.914248 \\
SYM9 & 44.314 & -53.6873 & 0.828558 & 6.865483 \\
SYM10 & 47.28782 & -50.3654 & 0.866303 & 6.891888 \\
\hline
\end{tabular}

Table 2. Comparison result SNR of de-noising signal 1 by daubechies wavelet

\begin{tabular}{lrccc}
\hline LEVEL & MAX $(\mathrm{dB})$ & MIN $(\mathrm{dB})$ & MEAN $(\mathrm{dB})$ & STD DEV $(\mathrm{dB})$ \\
\hline DB1 & 151.7097 & -28.3826 & 1.131428 & 7.903745 \\
DB2 & $\mathbf{5 6 . 1 0 4 1}$ & -49.6904 & $\mathbf{0 . 9 8 2 2 6 5}$ & $\mathbf{6 . 9 7 0 0 7 7}$ \\
DB3 & 56.09006 & -51.6243 & 0.978222 & 6.96467 \\
DB4 & 49.4397 & -51.6737 & 0.917533 & 6.872692 \\
DB5 & 49.61621 & -65.2694 & 0.812132 & 6.876263 \\
DB6 & 51.95115 & -49.5302 & 0.871651 & 6.899541 \\
DB7 & 46.61338 & -58.504 & 0.895262 & 6.890343 \\
DB8 & 43.70889 & -41.3558 & 0.796505 & 6.823182 \\
DB9 & 48.69122 & -49.2166 & 0.792285 & 6.914045 \\
DB10 & 42.94939 & $\mathbf{- 4 0 . 9 6 7 8}$ & 0.879292 & 6.882715 \\
\hline
\end{tabular}

Table 3. Comparison result SNR of de-noising signal 1 by coiflets wavelet

\begin{tabular}{lrccc}
\hline LEVEL & MAX $(\mathrm{dB})$ & MIN $(\mathrm{dB})$ & MEAN $(\mathrm{dB})$ & STD DEV $(\mathrm{dB})$ \\
\hline COIF1 & 52.44988 & -49.6667 & 1.05402 & 6.961058 \\
COIF2 & 47.99371 & -49.2107 & $\mathbf{0 . 9 6 8 6 2 7}$ & $\mathbf{6 . 9 3 1 1 9 2}$ \\
COIF3 & 44.82349 & -47.2696 & 0.892638 & 6.920543 \\
COIF4 & 44.98022 & $\mathbf{- 4 5 . 1 3 1 2}$ & 0.838424 & 6.824896 \\
COIF5 & $\mathbf{5 7 . 1 4 2 7 2}$ & -57.2028 & 0.789439 & 6.829048 \\
\hline
\end{tabular}

Referring to Table 1-3 and Figure 4-9, it can be concluded that all family first of mother wavelet cannot be chosen even it provides the highest SNR because the waveform are not significant to the original measured PD signal. In Symlets the best level is Sym4 base on result tabulated in Table 1 and the waveform in Figure 10. Its also indicates that the families from Sym2 to Sym4 is good to be use base on value of coefficient required either maximum, minimum, mean or standard deviation. In Daubechies, DB2 is selected from Table 2 because a majority of results in the table show the highest SNR and supported by significant waveform to signal in Figure 12. In Coiflets family, reading from Table 3 are scattered but the Coif 2 indicated a good family to be chosen since the readings in mean and standard deviations are the highest and supported by relevant waveform in Figure 11. Overall the Daubechies DB2 is selected as the best level from all mother wavelet based on the analysis has been performed. From this, de-noising process for measured PD signal analysis is performed under Daubechies 2 family with soft threshold so that a pure PD signal can be detected. 


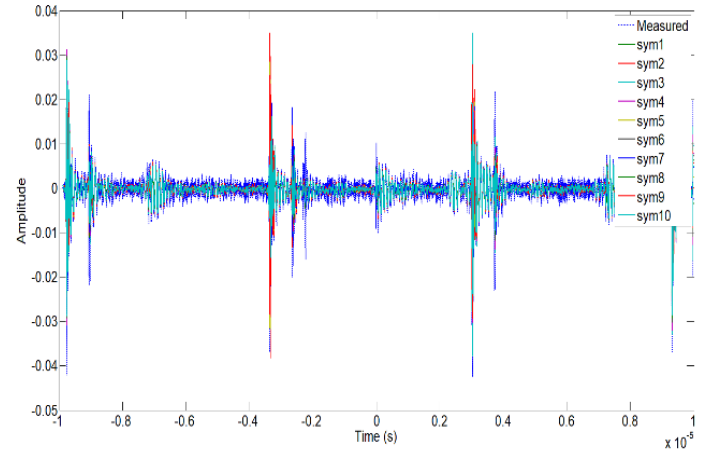

Figure 4. De-noised PD signal using symlets figure

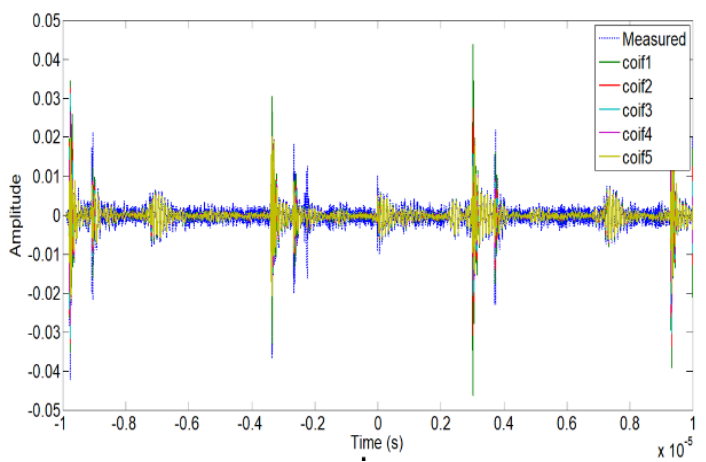

Figure 6. De-noised PD signal using coiflets

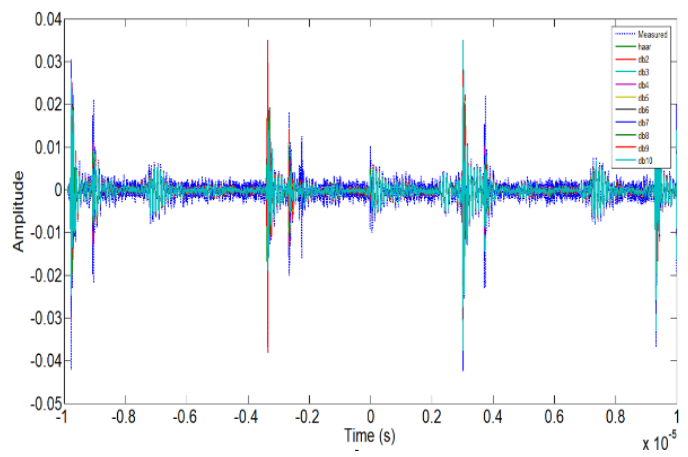

Figure 8. De-noised PD signal using daubechies

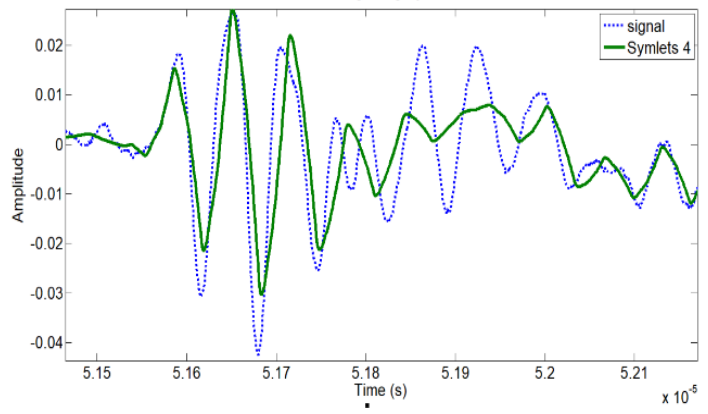

Figure 10. Zoomed de-noised using symlet 4

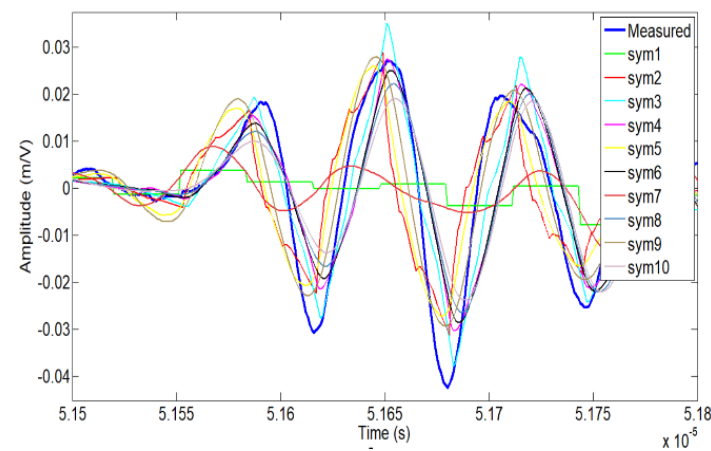

Figure 5. Zoomed de-noised PD signal using symlets

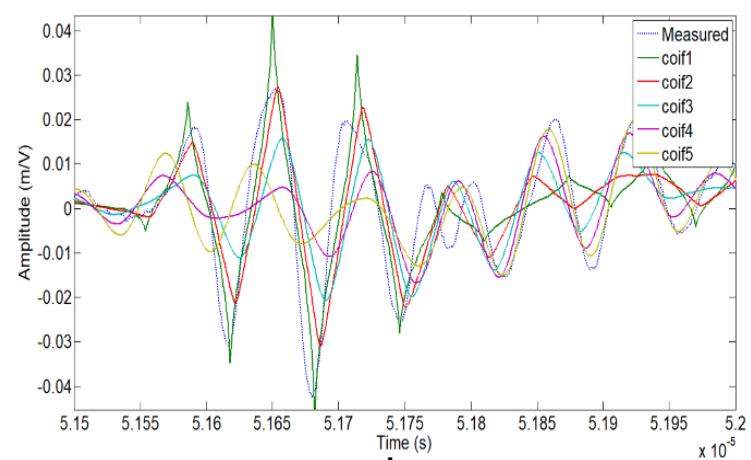

Figure 7. Zoomed de-noised PD signal using coiflets

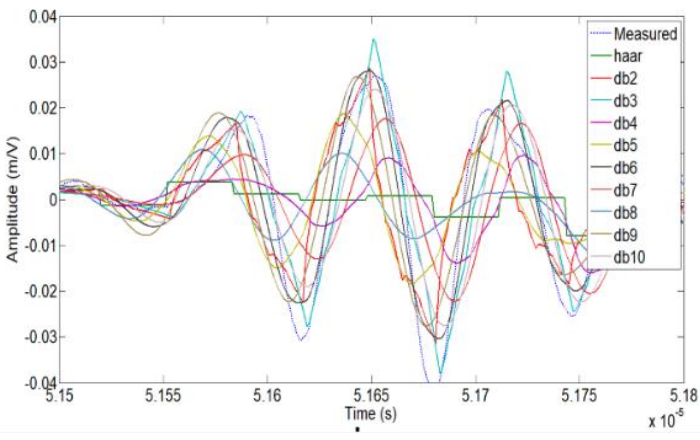

Figure 9. Zoomed de-noised PD signal using daubechies

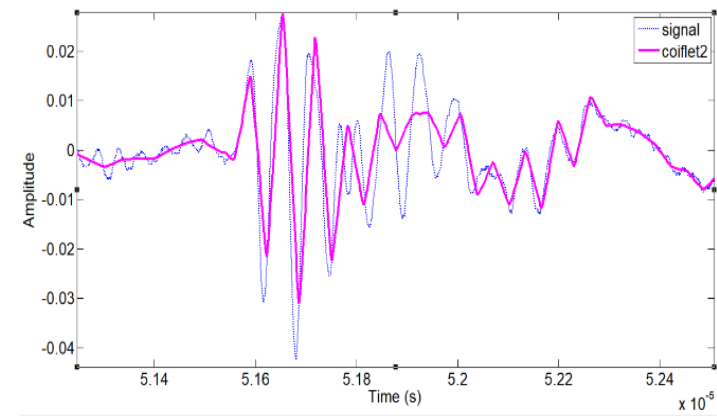

Figure 11. Zoomed de-noised using coiflets 2 


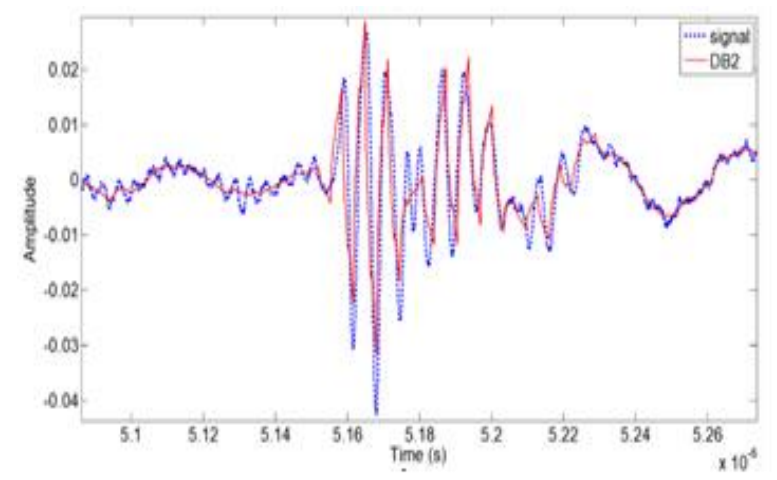

Figure 12. Zoomed de-noised PD Signal using daubechies 2

\section{CONCLUSION}

This paper presents wavelet based de-noising for on-site partial discharge measurement signal for medium voltage power cable. The wavelet transform as a powerful tool for noise removal was employed with real PD signal through the on-line measurement of a power cable. It was shown that PD signal in a power cable are comprise with noise can be easily separated by applying the de-noising technique, resulted in detection of pure PD signal. For the best selection wavelet from mother wavelet, the de-noising technique suggested that the optimum condition can be obtained by using Daubechies 2 which was supported by the experiment. Besides that, a detailed analysis should be performed which also include mean square error (MSE) calculation, signal energy and details correlation coefficient. Overall, the presents result show that Daubechies 2 can be used as mother wavelet for de-noising to obtain pure PD signal.

\section{ACKNOWLEDGEMENTS}

The authors gratefully acknowledge Universiti Malaysia Perlis (UniMAP) for the equipment support, Tenaga Nasional Berhad Research (TNBR) for the PD test reference on actual distribution utility, and to the Ministry of Higher Education for their support through the SLAB scheme.

\section{REFERENCES}

[1] D. A. Genutis, "Electrical Equipment Condition Assessment Using On-Line Solid Insulation Sampling," NETA WORLD Spring, pp. 1-5, 2006.

[2] A. B. J. M. Driessen, J. Van Duivenbode, and P. A. A. F. Wouters, "Partial Discharge Detection for Characterizing Cable Insulation under Low and Medium Vacuum Conditions," pp. 306-315, 2018.

[3] J. P. Preis, Kurt, I.Ticar, "Partial discharges in insulation of medium voltage systems," Int. J. Comput. Math. Electr. Electron. Eng., vol. 20, no. 2, pp. 473-481, 2001.

[4] Muzamir Isa, "Partial Discharge Location Technique for Covered- Conductor Overhead Distribution Lines," Aalto University, 2013.

[5] A. Mukhtaruddin, M. Isa, M. R. Adzman, S. I. S. Hasan, M. N. K. H. Rohani, and C. C. Yii, "Techniques on partial discharge detection and location determination in power transformer," $20163 \mathrm{rd}$ Int. Conf. Electron. Des. ICED 2016, pp. 537-542, 2017.

[6] L. Renforth, R. Giussani, M. T. Mendiola, and L. Dodd, "On-Line Partial Discharge Insulation Condition Monitoring of Complete High Voltage Networks," IEEE Trans. Ind. Appl., vol. 9994(c), pp. 1-9, 2018.

[7] P. C. J. M. Van Der Wielen, On-line Detection and Location of Partial Discharges in Medium-Voltage. 2005.

[8] C. P. Dautov and M. S. Ozerdem, "Wavelet transform and signal de-noising using Wavelet method," 26th IEEE Signal Process. Commun. Appl. Conf. SIU 2018, pp. 1-4, 2018.

[9] A. Kyprianou and G. E. Georghiou, "Wavelet Packet De-noising for On-Line Partial Discharge Detection in High Voltage Systems," Proc. 2005 IEEE Int. Symp. on, Mediterrean Conf. Control Autom. Intell. Control. 2005., pp. 1184-1189, 2005.

[10] F. Chang, W. Hong, T. Zhang, J. Jing, and X. Liu, "Research on wavelet de-noising for pulse signal based on improved wavelet thresholding," Proc. - 2010 1st Int. Conf. Pervasive Comput. Signal Process. Appl. PCSPA 2010(4), pp. 564-567, 2010.

[11] M. Ghaffarian, M. Vakilian, V. Parvin, and A. Ghaedi, "Investigation of Online Detected Partial Discharges in power transformer," pp. 1-6, 2008.

[12] A. Kraetge, S. Hoek, R. Hummel, and M. Krüger, "De-noising approaches for partial discharge measurements - A comparison of methods and their practical application," 2012 IEEE International Conference on Condition Monitoring and Diagnosis, . 538-541, 2012. 
[13] M. Jayakrishnan, "Application of Modified Wavelet Packet Transform for De-noising during Partial Discharge Measurement on Power Cables," 2017 3rd International Conference on Condition Assessment Techniques in Electrical Systems (CATCON) pp. 2-7, 2018.

[14] P. Ray, "Optimum Wavelet Bases Selection for Wavelet Based De-Noising In Partial Discharge Measurement," 2013 IEEE Conf. Inf. Commun. Technol., no. Ict, pp. 1110-1113, 2013.

[15] S. H. K. Hamadi et al., "Modelling of partial discharge signal and noise interference using labview," IEEE Student Conf. Res. Dev. Inspiring Technol. Humanit. SCOReD 2017 - Proc., vol. 2018-Janua, pp. 451-455, 2018.

[16] C. C. Yii, M. N. K. H. Rohani, M. Isa, and S. I. S. Hassan, "Multi-end PD location algorithm using segmented correlation and trimmed mean data filtering techniques for MV Underground Cable," IEEE Trans. Dielectr. Electr. Insul., vol. 24(1), pp. 92-98, 2017.

[17] J. C. Chan, H. Ma, T. K. Saha, and C. Ekanayake, "A novel level-based automatic wavelet selection scheme for Partial Discharge measurement," 2012 22nd Australas. Univ. Power Eng. Conf. "Green Smart Grid Syst., pp. 1-6, 2012.

[18] G. Luo and D. Zhang, "Application of wavelet transform to study partial discharge in XLPE sample," Power Eng. Conf. 2009. 2009.

[19] J. Too, A. R. Abdullah, N. M. Saad, N. M. Ali, and H. Musa, “A Detail Study of Wavelet Families for EMG Pattern Recognition,” International Journal of Electrical \& Computer Engineering., vol. 8(6), pp. 4221-4229, 2018.

[20] M. N. K. H. Rohani et al., "Evaluation of Rogowski coil sensor performance using EMTP-ATP software," 2016 3rd Int. Conf. Electron. Des. ICED 2016, pp. 446-451, 2017.

[21] M. N. K. H. Rohani, M. Isa, M. Syahril, C. C. Yii, A. S. Rosmi, and B. Ismail, "Sigma-Delta ADC Topology Implementation Based on Partial Discharge Detection using Rogowski Coil Sensor," J. Phys. Conf. Ser., vol. 1019(1), 2018.

[22] M. N. K. H. Rohani et al., "Geometrical Shapes Impact on the Performance of ABS-Based Coreless Inductive Sensors for PD Measurement in HV Power Cables," IEEE Sens. J., vol. 16(17), pp. 6625-6632, 2016.

[23] J. Hu, D. Liu, Q. Liao, X. Han, and Y. Yan, "Analysis of substation noise prediction based on equivalent source method," Asia-Pacific Power Energy Eng. Conf. APPEEC, vol. 2015-March, no. March, pp. 1-5, 2014.

[24] M. H. Jopri, A. R. Abdullah, T. Sutikno, M. Manap, M. R. A. Ghani, and M. R. Yusoff, "A Critical Review of Time-frequency Distribution Analysis for Detection and Classification of Harmonic Signal in Power Distribution System," International Journal of Electrical \& Computer Engineering, vol. 8(6), pp. 4603-4618, 2018.

[25] C. Kane, B. Lease, A. Golubev, and D. Ph, "Practical Experiences of On-Line Partial Discharge Measurements on a Variety of Medium Voltage," IEEE Trans. Ind. Appl., vol. 35(6), 1999.

[26] W. H. S. I.Shim, J.J.Saraghan, "Digital Signal Processing Applied to the Detection of Partial Discharge : An Overview," IEEE Electr. Insul. Mag., vol. 16(3), pp. 6-12, 2000.

[27] N. A. Yusoff, M. Isa, H. Hamid, M. R. Adzman, M. Nur, and K. Hafizi, "De-noising Technique for Partial Discharge Signal : A Comparison Performance between Artificial Neural Network, Fast Fourier Transform and Discrete Wavelet Transform," 2016 IEEE International Conference on Power and Energy (PECon), 2018.

[28] S. Sriram and K. M. M. Prabhu, "Signal De-noising Techniques for Partial Discharge Measurements," IEEE Trans. Dielectr. Electr. Insul., vol. 12, pp. 1182-1191, 2005.

\section{BIOGRAPHIES OF AUTHORS}

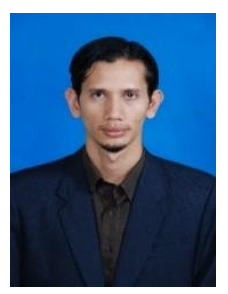

Ahmad Zaidi Bin Abdullah was born in Kedah, Malaysia in 1981. He received his B.Eng (Hons.) degree in Industrial Electronic Engineering from University Malaysia Perlis and M.Eng in Electrical Power from University Tenaga Nasional. He is currently pursuing a $\mathrm{PhD}$ at Universiti Malaysia Perlis. He is a member of Board Engineer Malaysia and Institute Engineer Malaysia. His main research interest are high-voltage engineering, power transformer and partial discharge online monitoring.

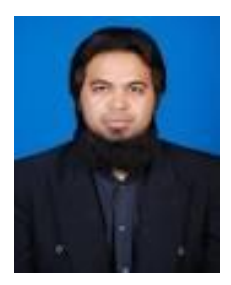

Muzamir Bin Isa was born in Perlis, Malaysia in 1979. He received his B.Eng (Hons.) degree in Electrical Engineering from University Teknologi Malaysia, M.Eng in Electrical Power from University Tun Hussein Onn Malaysia, and PhD in Power System and High Voltage Engineering from Aalto University, Finland. He is a member of the Board Engineer Malaysia. His main research interest are high-voltage engineering, power transformer and partial discharge on-line monitoring. 

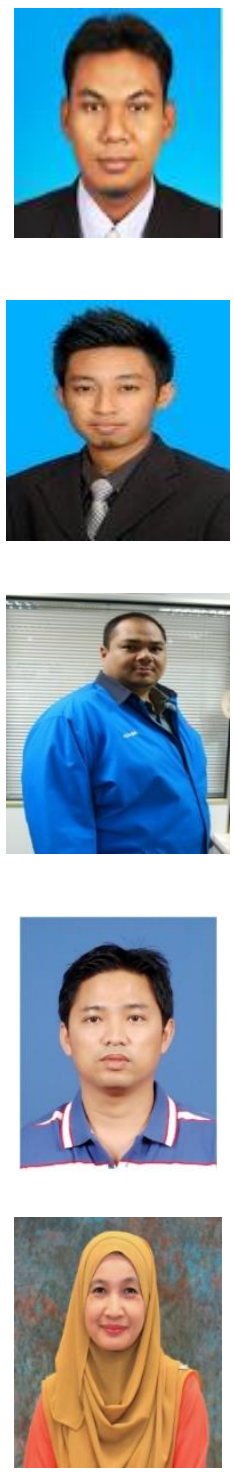

Syahrun Nizam Bin Arshad was born in Kedah, Malaysia in 1984. He received his B.Eng (Hons.) degree in Electrical Engineering and M.Eng in Electrical Power from University Teknologi Malaysia, and PhD in Lightning from Universiti Putra Malaysia. He is a member of the Board of Engineer Malaysia. His main research interest are high-voltage engineering, grounding and lightning.

Mohamad Nur Khairul Hafizi Rohani was born in Johor, Malaysia, in 1989. He received the B.Eng. (Hons.) degree in industrial electronic engineering and $\mathrm{PhD}$ from the University Malaysia Perlis, Malaysia, in 2013. His research interests is the design of sensors for online condition components based on partial discharge measurement.

Huzainie Shafi Abd. Halim received his B. Eng. (Hons) in Electronic and Electrical Engineering from the University of Portsmouth, UK in 1997. Upon graduation, he worked with ABB (Malaysia) Transmission \& Distribution as a Protection Design Engineer. Since 1998, he has been employed by TNB Research as a Research Engineer with Product Design and Prototyping Group. In 2000 he joined the High Voltage Diagnostic Research Group in TNB Research. He obtained his M. Eng. in Electrical Engineering from University Tenaga Nasional in 2010. He is a member of Board Engineer Malaysia and Institute Engineer Malaysia. His main focus area lies with cable insulation diagnostic and measurements. Presently he is a Technical Expert of Power Cable in TNB Research.

Ayob Nazmi Bin Nanyan was born in Langkawi, Malaysia, in 1982. He received a B.Eng. (Hons.) degree in Electrical System Engineering and a Master of Science in Electrical from University Malaysia Perlis, Malaysia. His research interests is the design of sensors for online condition components based on over voltage measurement.

Haziah Binti Abd Hamid received the first and master's degree in electrical engineering from Universiti Teknologi Malaysia (UTM) in 1999 and 2002 respectively. Then she received the Ph.D in Electrical and Electronic Engineering from Cardiff University, UK in 2012. She is a senior lecturer and currently holding the position of Dean at the School of Electrical System Engineering, Universiti Malaysia Perlis, Malaysia. Her main research interests are in high voltage transients, insulation system, and earthing system. 